\title{
EL CAMBIO DE PARADIGMA DE LA GESTIÓN DEL DRENAJE URBANO DESDE LA PERSPECTIVA DEL PLANEAMIENTO. UNA PROPUESTA METODOLÓGICA
}

\author{
$M^{a}$ Isabel Rodríguez-Rojas ${ }^{1}$ \\ $M^{\mathrm{a}}$ Mar Cuevas-Arrabal ${ }^{2}$ \\ Begoña Moreno Escobar ${ }^{3}$ \\ Germán Martínez Montes ${ }^{4}$
}

Universidad de Granada

\section{RESUMEN}

La impermeabilización de la ciudad, fruto de un modelo de ocupación urbana intensivo, ha generado un aumento de los volúmenes de escorrentía que ha justificado la ampliación continua y siempre insuficiente de las redes de saneamiento, consolidando un modelo de gestión del drenaje insostenible. En los últimos años se está demandando un tratamiento de las aguas pluviales más acorde con los principios medioambientales, en el que la planificación sea el marco de referencia para el control del sellado del suelo y la mejora de la sostenibilidad urbana. En este artículo se presenta una propuesta metodológica para la aplicación de este nuevo modelo denominado 'diseño urbano sensible al agua' (DUSA), definiendo sus elementos principales y su integración en el planeamiento urbano.

Palabras clave: planeamiento urbano, diseño urbano sensible al agua, sistema urbano de drenaje sostenible, SUDS, aguas pluviales, gestión del agua.

Fecha de recepción: noviembre 2015.

Fecha de aceptación: noviembre 2016.

1 Departamento de Urbanismo y Ordenación del Territorio. Universidad de Granada, mabel@ugr.es

2 Departamento de Urbanismo y Ordenación del Territorio. Universidad de Granada, marcuevas@ugr.es

3 Departamento de Ingeniería de la Construcción y Proyectos de Ingeniería. Universidad de Granada, bgmoreno@ ugr.es

4 Departamento de Ingeniería de la Construcción y Proyectos de Ingeniería. Universidad de Granada, gmmontes@ugr.es 


\section{ABSTRACT}

The impermeabilization of cities, which is the result of a model of intensive urban occupation, has generated an increase in the volume of run-off, which has justified the continued and constantly insufficient expansion of sanitation networks, establishing an unsustainable drainage management model. In recent years, there has been a demand for rainwater treatment which is more consistent with environmental principles, in which planning would be the frame of reference for the control of soil sealing and improvement in urban sustainability. This article presents a methodological proposal for the application of this new model called 'water sensitive urban design'(WSUD), defining its main elements and its integration in urban planning.

Keywords: urban planning, water sensitive urban design, sustainable urban drainage system, SUDS, rainwater, water management.

\section{INTRODUCCIÓN Y OBJETO DEL TRABAJO}

Los grandes cambios socioeconómicos iniciados en Europa tras la Revolución Industrial, han generado una enorme densificación y expansión de las ciudades en muchos casos descontrolada (Antrop, 2004; García et. al, 2014; Blum et. al, 2004), que se ha traducido en una tasa de cambio de suelo de rústico a urbano superior a la tasa de crecimiento demográfico (Lavalle et. al, 2001; Montanarella, 2007; Van-Camp et. al, 2004). En Europa, por ejemplo, entre 1980 y 2000 la extensión de las zonas edificadas se incrementó en un 20\% mientras que la densidad de población sólo en un $10 \%$ (Fig. 1). Este fenómeno, originado fundamentalmente por la consolidación de un modelo de desarrollo urbano de baja densidad (EEA, 2006), en el que la sociedad se ha expandido sobre el campo sustituyendo su condición rural por urbana (Monclús, 1998), está ocasionando grandes desigualdades (Ferras, 2000), el despilfarro energético y de recursos necesarios para dotar de servicios a una población desconcentrada (Sierra Club, 2000), la ocupación indiscriminada de grandes lotes de suelo rural (Bengston et al., 2004) y numerosos impactos ambientales (Prud'home y Lee, 1999; Gobster et al., 2004), entre ellos la impermeabilización de las superficies o 'sellado del suelo'5 (Houšková et. al, 2008; Prokop et. al, 2011; Tóth et. al, 2008). Este proceso está teniendo graves consecuencias sobre el territorio y la ciudad, produciendo la pérdida de biodiversidad y hábitats (Carsjens y Van Lier, 2002; Gibb y Hochuli, 2002), la destrucción de suelos agrícolas y forestales (Morello et al., 2000; Lawrence et al., 2002; Chen et al., 2003; Imhoff et al., 2004), la degradación del paisaje (SWD 101, 2012), la modificación del clima urbano o efecto "isla de calor' ${ }^{6}$ (Gartland, 2008) (Fig. 2) y la alteración del ciclo hidrológico (Imhoff et al., 2004).

5 La importancia de este fenómeno es tal, que en 2012 la Comisión Europea creó un grupo de trabajo específico para analizar las consecuencias del 'sellado del suelo' en Europa, elaborando un documento denominado "Directrices sobre las mejores prácticas para limitar, mitigar o compensar el sellado del suelo”, que marca las líneas de acción para políticas, planes y programas en los diferentes estados miembros de la UE en materia de suelos (SWD 101, 2012).

6 Incremento de temperatura en las zonas urbanas producido por la destrucción de la refrigeración natural del suelo, la absorción de energía solar por los techos y superficies de asfalto oscuro, el calor residual generado por la industria, los sistemas de refrigeración y el tráfico (Arnfield, 2003). Genera importantes problemas de 
Figura 1

EVOLUCIÓN DE LA SUPERFICIE CONSTRUIDA Y DENSIDAD DE POBLACIÓN EN EUROPA

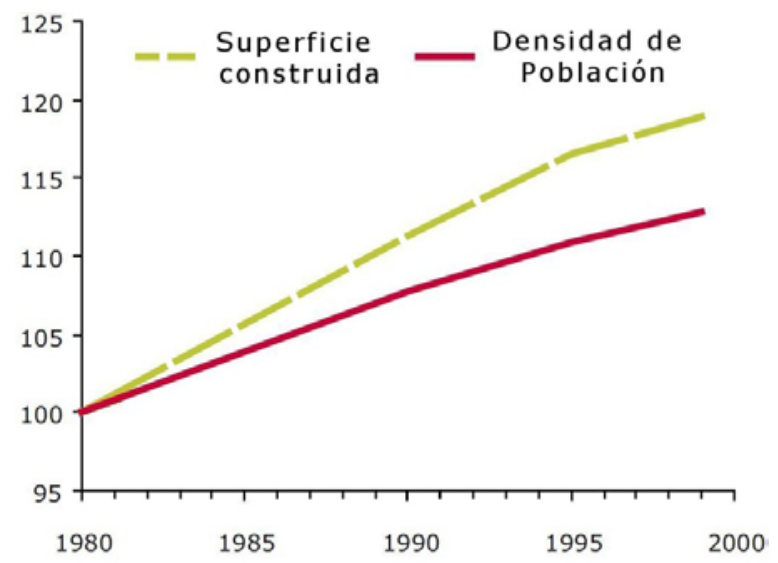

Fuente: Modificado de EEA, 2006.

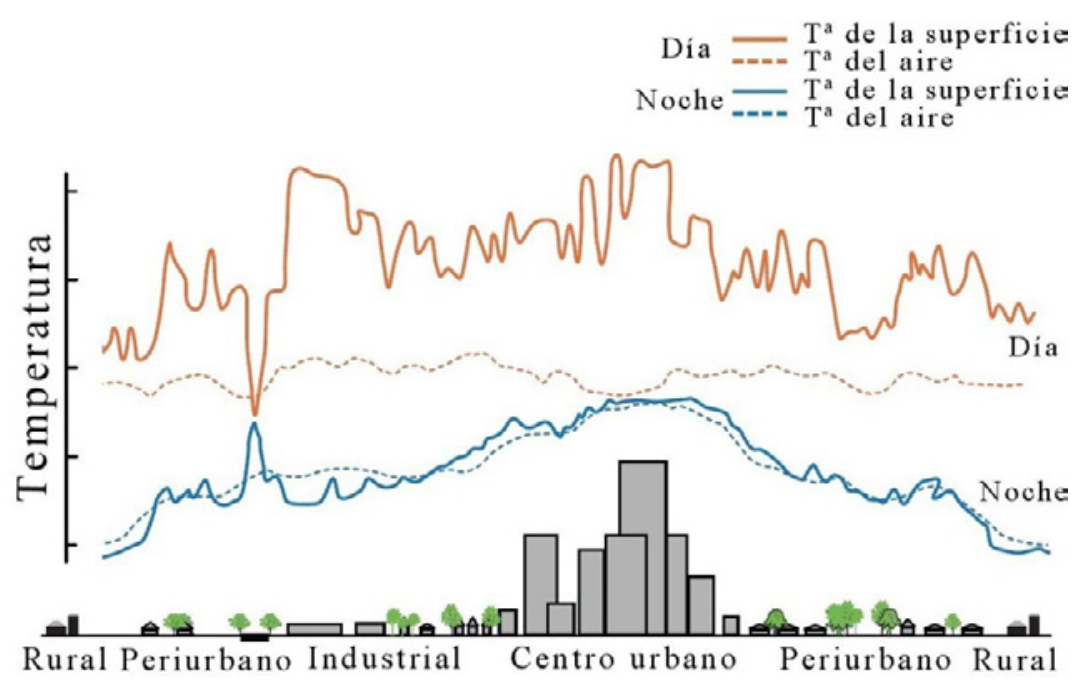

Fuente: Modificado de EPA, 2008.

salud a los ciudadanos debido a la acumulación de contaminantes y a la falta de renovación del aire (Bolund et. al, 1999), así como un incremento de la demanda energética por la necesidad de refrigeración (Santamouris et. al, 2001) y una disminución del confort térmico en la ciudad por la alteración de la humedad y velocidad del viento (Elsayed, 2012). 
El principal efecto de la impermeabilización de las superficies sobre el ciclo hidrológico urbano ha sido sin duda la disminución de la capacidad de infiltración y retención del suelo (Fig. 3). Esta alteración ha producido un incremento de la escorrentía superficial que está colapsando las redes de saneamiento tradicionales ${ }^{7}$ (Ren et al., 2013), la mayoría de las cuales fueron proyectadas en los ensanches de finales del siglo XIX y han sido ampliadas durante todo el siglo XX hasta el límite de sus posibilidades. Los crecientes volúmenes de drenaje generados por los nuevos desarrollos urbanos han provocado un incremento de las inundaciones en los últimos años que está teniendo consecuencias catastróficas sobre todo en los entornos fluviales (Burghardt et. al, 2004; EEA, 2010), donde las crecidas naturales de los ríos se han visto incrementadas por el aporte extra de escorrentía procedente de las ciudades (Conesa et al., 2003; García, 2004). Este fenómeno, ya de por sí grave a día de hoy, empeorará previsiblemente en los próximos años por el incremento de intensidad de las lluvias que prevén los actuales modelos de cambio climático $^{8}$ (Olsson et. al, 2009).

Esta situación pone de manifiesto la necesidad de generar un cambio de paradigma en la gestión del drenaje urbano que termine con un modelo insostenible, basado en la ampliación continua y siempre insuficiente de las redes de saneamiento (Olsson et. al, 2009), y que integre la planificación urbana como una herramienta para el control de la impermeabilización del suelo y la mejora de la sostenibilidad urbana.

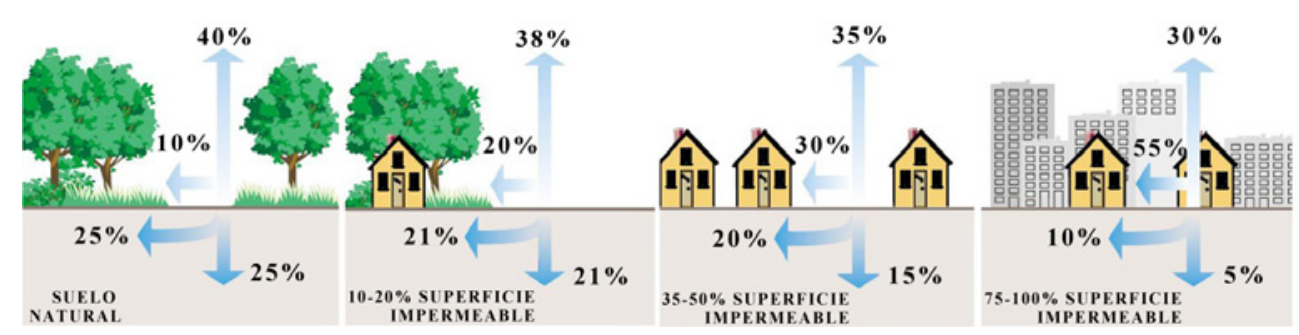

Fuente: Modificado de Hausmann, 2014.

En este sentido, desde los años noventa se está desarrollando una estrategia más acorde con estos principios mediante la utilización de los denominados 'sistemas urbanos de drenaje sostenible' o 'SUDS” (CIRIA C697, 2007; MWB, 2013), cuyo objetivo es resolver los problemas ambientales derivados del sellado del suelo, especialmente los relacionados con el ciclo del agua (Prokop et. al, 2011). También conocidos como BMP (best management practice) o LID (low impact development), estos sistemas recogen el agua de lluvia, la transportan y almacenan durante el máximo de tiempo posible con objeto de ralentizarla,

\footnotetext{
7 Redes unitarias que transportan conjuntamente el agua pluvial y residual (Catalá, 1992).

8 Algunos modelos (MikeSHE, MOUSE,..) predicen lluvias que generarán aumentos de los caudales de agua de escorrentía entre un 20 y un 40\%, según el escenario planteado (Semadeni-Daviesa et. al, 2009).

9 Iniciales del término original en inglés 'sustainable urban drainage systems'.
} 
y después la infiltran y reutilizan en usos no consuntivos o simplemente para la recarga de acuíferos (CIRIA C697, 2007), recuperando en la medida de lo posible el ciclo hidrológico del agua en la ciudad (Rodríguez-Rojas et al., 2015).

Países como EE.UU., Reino Unido, Canadá, Francia o Australia utilizan los SUDS desde hace más de una década (CIRIA C521, 2000), en la mayoría de ocasiones como una herramienta para mitigar a posteriori los efectos del sellado del suelo, por lo que los resultados obtenidos, a pesar de haber sido muy positivos no han sido definitivos. Este es el caso de algunos proyectos estadounidenses ('Millers creek rain water project', 'Northern kentucky sanitation district', 'Waltham woods') que lograron reducir importantes volúmenes de escorrentía en un inicio (40\%, 90\% y 100\% respectivamente) (Asla, 2015), pero vieron disminuida su eficacia con el paso del tiempo debido a la aparición de nuevas de superficies impermeables. Estas y otras experiencias ponen de manifiesto la necesidad de llevar a cabo una planificación urbana coordinada que defina las estrategias necesarias para controlar el impacto del sellado del suelo sobre el ciclo hidrológico en la ciudad.

Respondiendo a este requerimiento, en los últimos años se ha planteado un nuevo modelo integral agua-suelo denominado 'diseño urbano sensible al agua' ${ }^{10}$ (DUSA), que se define como 'la integración del planeamiento urbano en la gestión, protección y conservación del ciclo urbano del agua, de forma que se asegure una gestión del agua sensible a los procesos hidrológicos y ecológicos' (COAG, 2004). Este modelo supone un avance respecto de los SUDS, pues más allá de la intervención puntual que implica la implantación de estos sistemas, plantea una forma de ocupación del territorio más sostenible y acorde con los procesos hidrológicos, incorporando el diseño de los elementos urbanos (calles, edificios, espacios públicos, aparcamientos ,...) como una valiosa oportunidad para restaurar la calidad ambiental de la ciudad (Rodríguez-Rojas et. al, 2014). Para su aplicación, el planificador debe tener en cuenta tres principios básicos:

- Proyectar superficies urbanas que potencien al máximo la infiltración del agua pluvial.

- Favorecer la retención de la escorrentía en aquellos lugares donde sea posible diseñando nuevos espacios para tal efecto si fuera necesario.

- Buscar y crear oportunidades para la reutilización del agua infiltrada y/o retenida.

La aplicación de estos principios generará numerosos e importantes beneficios ambientales, sociales y económicos para la ciudad (Fig. 4), haciendo más visibles los procesos hidrológicos y logrando una mayor implicación del ciudadano en su manejo y conservación, lo que se traducirá en una mejor gestión de este valioso recurso.

Existen en la actualidad muy pocas referencias que trabajen sobre este modelo (CIRIA C687, 2010), predominando todavía los manuales especializados en SUDS (CIRIA C697, 2007; SWD 101, 2012; MWB, 2013), que muestran con detalle el proceso de ejecución técnica de estos sistemas pero no tratan los aspectos relacionados con su integración en el planeamiento. Esta carencia deja patente la necesidad de desarrollar guías específicas dirigidas a

10 Traducción del término original 'water sensitive urban design' (WSUD), acuñado por la 'National Water Commission' en el 'Council of Australian Governments' de 2004. 
urbanistas que faciliten y potencien la aplicación de este nuevo modelo en el ámbito urbano. De este modo, el principal objetivo de este artículo es aportar una herramienta metodológica que defina e integre los requerimientos del DUSA en el proceso de planificación, contribuyendo así al desarrollo de proyectos sensibles al agua y en consecuencia, a la mejora de la calidad ambiental de las ciudades y de la sostenibilidad de la gestión del recurso hídrico.

Figura 4

OBJETIVOS Y BENEFICIOS DEL DISEÑO URBANO SENSIBLE AL AGUA

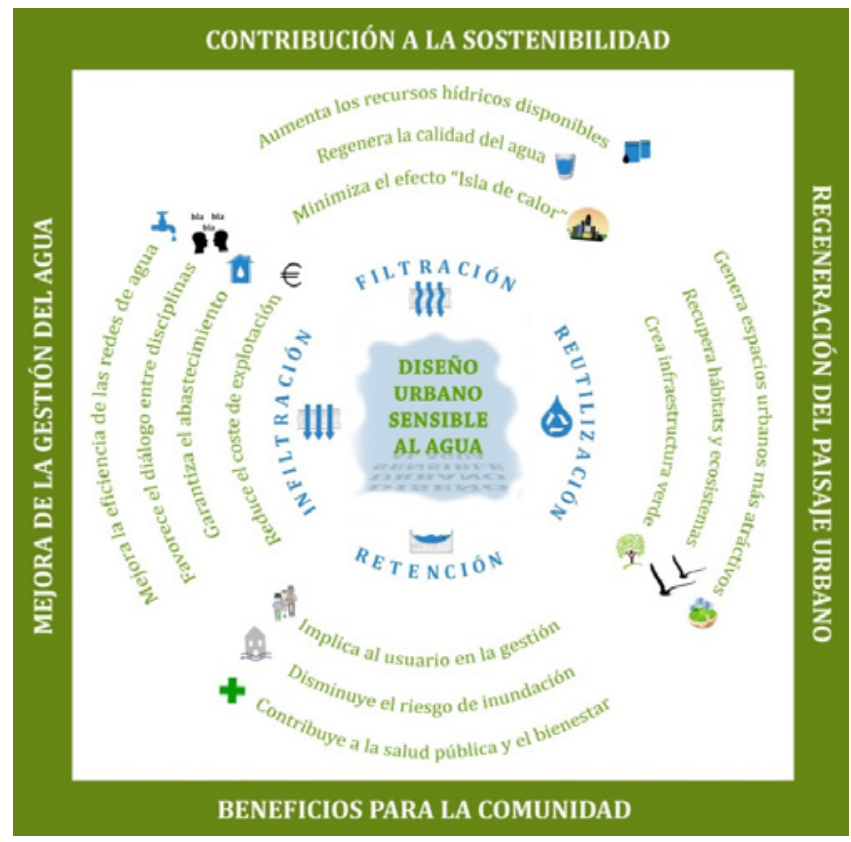

Fuente: elaboración propia.

\section{METODOLOGÍA DE PLANEAMIENTO PARA EL DISEÑO URBANO SENSIBLE AL AGUA}

Para facilitar la incorporación del DUSA en el proceso de planeamiento debe procurarse que éste se inserte en todas sus etapas, asegurando de este modo la viabilidad, rentabilidad y beneficios del mismo. Las metodologías empleadas en el planeamiento desarrollan diferentes fases de trabajo según el autor, aunque en todas ellas pueden observarse de un modo u otro las siguientes (Rebstock et al., 2011): preparación del plan (diálogos previos con los agentes implicados y análisis y diagnóstico de la situación existente), diseño y evaluación de las propuestas estratégicas, implementación o ejecución de los proyectos que desarrollan las líneas de trabajo definidas y monitorización o seguimiento de los resultados obtenidos. A continuación se muestra una propuesta metodológica para la integración del DUSA en esta estructura, de modo que el agua sea una exigencia y una oportunidad más en el proceso de planeamiento (Fig. 5). 


\section{II.1. Diálogos previos}

En la primera fase del planeamiento debe construirse un proceso de diálogo con los diferentes agentes sociales implicados en el proyecto (comunidades de vecinos, asociaciones, centros educativos...), con el objetivo de dar a conocer el DUSA y explicar su importancia para la mejora de la calidad ambiental de la ciudad y del bienestar del ciudadano. Esta cuestión es fundamental para lograr la implicación de la comunidad en el mantenimiento de los proyectos que lo requieran y asegurar así el éxito de las propuestas ${ }^{11}$.

Figura 5

METODOLOGÍA PARA LA INTEGRACIÓN DEL DUSA EN EL PLANEAMIENTO

\section{DISEÑO URBANO SENSIBLE ALAGUA}

\begin{tabular}{|c|c|}
\hline \multicolumn{2}{|c|}{ DIÁLOGOS PREVIOS } \\
\hline Agentes sociales & Integración de disciplinas \\
\hline Agentes politicos y técnicos & \\
\hline \multicolumn{2}{|c|}{ ANÁLISIS Y DIAGNÓSTICO } \\
\hline \multicolumn{2}{|c|}{ Medio fisico : topografia, geologia, climatologia } \\
\hline Medio urbano & Medio hidrológico-hidráulico \\
\hline Trama urbana: 'calles rio'y 'calles lago' & Red de drenaje \\
\hline Permeabilidad: tipos de superficies & Cálculo de caudales \\
\hline Espacios degradados & Zonas inundables \\
\hline Zonas verdes $\mathrm{y}$ áreas multifuncionales & \\
\hline
\end{tabular}

\begin{tabular}{|c|c|}
\hline \multicolumn{2}{|c|}{ PROPUESTAS } \\
\hline \multicolumn{2}{|c|}{ Creación de corredores azules y conexión con espacios verdes y áreas de infiltración } \\
\hline \multicolumn{2}{|c|}{ Incremento de las superficies permeables } \\
\hline \multicolumn{2}{|c|}{ Integración de los SUDS en la trama urbana existente y propuesta' } \\
\hline \multicolumn{2}{|c|}{ Diseño del plan de mantenimiento e implicación de los usuarios } \\
\hline \multicolumn{2}{|c|}{ EVALUACIÓN } \\
\hline Presentación y discusión & Valoración de resultados obtenidos \\
\hline \multirow{4}{*}{$\begin{array}{l}\text { Revisión de las propuestas con los agentes } \\
\text { sociales, politicos y técnicos }\end{array}$} & Disminución del riesgo de inundación \\
\hline & Reducción y reutilización del agua pluvial \\
\hline & Incremento de superficies permeables \\
\hline & Disminución de la temperatura en la ciudad \\
\hline \multicolumn{2}{|c|}{ MONITORIZACIÓN } \\
\hline \multicolumn{2}{|c|}{ Revisión de los resultados obtenidos } \\
\hline
\end{tabular}

Fuente: elaboración propia. ${ }^{1}$ Ver Cuadros 1 y 2.

11 Algunas experiencias previas han fracasado por no contar con la implicación del vecindario en el seguimiento de los proyectos directamente relacionados con el ámbito de la vivienda y sus proximidades (cubiertas verdes, depósitos de retención domésticos, jardines de lluvia, etc...) (MWB, 2013). 
De igual forma, deben realizarse encuentros con los agentes políticos y técnicos (autoridades local y regional, órganos de gestión del agua y del suelo, entidades gestoras de las redes del agua,...) con el fin de no incurrir en contradicciones normativas y de solape con otros proyectos.

En paralelo a este proceso de discusión es importante integrar en el equipo del proyecto todas las disciplinas necesarias para tratar la complejidad del problema urbano en su relación con el agua (urbanismo, geografía, ingeniería, ciencias ambientales, paisajismo, legislación, sociología,...). La multidisciplinariedad asegurará la transversalidad de las propuestas realizadas satisfaciendo todas las necesidades.

\section{II.2. Análisis y diagnóstico}

Una vez finalizados los diálogos previos comienza la fase de evaluación de la situación actual del ámbito de trabajo. Para ello se llevará a cabo un análisis y diagnóstico de la problemática existente, siendo necesario conocer con detalle las características físicas del lugar en su relación con el agua (topografía, geología, climatología,...), el medio urbano (calles, superficies, espacios verdes...) y sus características hidrológico-hidráulicas (red de drenaje, caudales, zonas inundables...) (Fig. 6). Esta fase resulta crucial para la integración del DUSA en el planeamiento, pues define las principales restricciones y potencialidades de diseño asociadas al agua que deberán tenerse en cuenta en el posterior desarrollo de las propuestas.

Respecto del medio urbano, es necesario analizar con detenimiento la trama viaria propuesta o existente en su relación con la topografía, con el fin de localizar las calles que no poseen suficiente pendiente para evacuar el agua de drenaje por sí mismas y que por tanto pueden inundarse (las denominaremos 'calles lago' ${ }^{12}$ ), así como aquellas que tienen una elevada pendiente pudiendo generar velocidades de escorrentía peligrosas (las denominaremos "calles río" ${ }^{13}$ ).

La segunda variable a estudiar es la permeabilidad, pues ésta afecta de forma directa a la infiltración del agua pluvial ${ }^{14}$. Debe llevarse a cabo un estudio específico de los usos del suelo existentes con el fin de determinar qué tipo de superficies se pueden proyectar y/o modificar en el área de intervención, de forma que se favorezca todo lo posible la infiltración de la escorrentía y se aumente al máximo el tiempo de retención de la misma. En este sentido, deben localizarse también aquellos espacios urbanos degradados que puedan ser rehabilitados y/o restaurados mediante el reacondicionamiento de sus superficies o a través de la implantación de algún SUDS proyectado a tal efecto (humedales, cuentas verdes, áreas de bio-retención ...). Por último, es necesario localizar las zonas verdes y áreas multifuncionales que puedan ser utilizadas como espacios de recogida y almacenamiento temporal de agua pluvial para su posterior infiltración y retención, pues su función será crucial para evitar inundaciones.

12 Se considera que para la evacuación del drenaje por gravedad es necesaria una pendiente mínima del 2-3\% (Catalá, 1992).

13 La escorrentía originada en calles con pendientes mayores al 20\% puede generar erosión y riesgo para la vida humana en caso de inundaciones (Minvu-Dictuc, 1996).

14 Algunos estudios muestran que las áreas edificadas pueden ser hasta 4 veces más impermeables que las zonas verdes o las parcelas sin edificar (Burghardt et. al, 1993). Es recomendable dejar al menos un 50\% de espacio sin pavimentar para mantener tasas satisfactorias de infiltración (Technische Commissie Bodem, 2010). 
En relación al medio hidrológico-hidráulico, para llevar a cabo un planeamiento urbano en condiciones de seguridad es necesario conocer con detalle la red de drenaje superficial existente, el caudal que circulará por ellos y las zonas inundables que pueden generarse ${ }^{15}$. De esta forma, las propuestas deberán ir encaminadas a aumentar el tiempo de recorrido del agua por la red de calles, a disminuir el volumen de agua circulante en su superficie y a reducir la cantidad de áreas afectadas por las inundaciones y la frecuencia de las mismas.

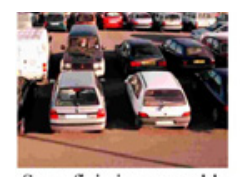

Superficie impermeable

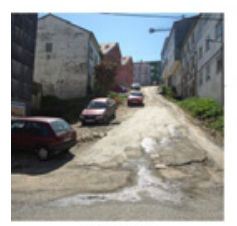

Calle rio

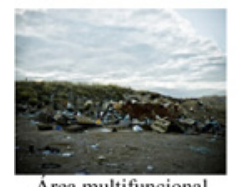

Área multifuncional

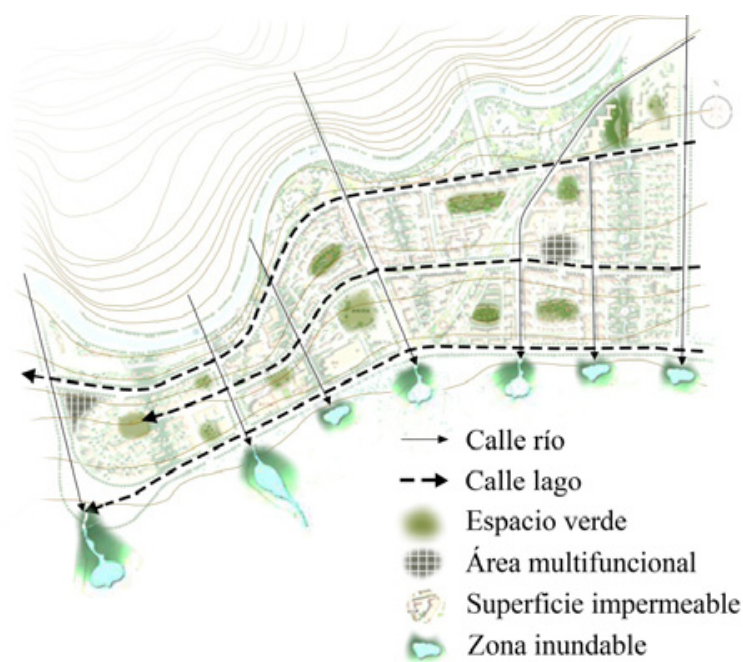

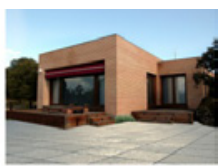

Superficie impermeable

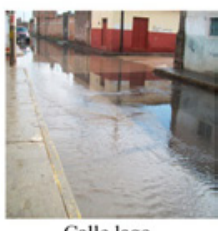

Calle lago

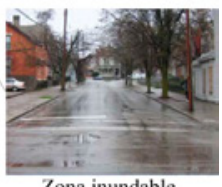

Zona inundable

Fuente: elaboración propia.

\section{II.3. Propuestas}

En la fase de intervención deben establecerse relaciones solidarias entre los medios urbano e hidrológico-hidráulico analizados, de forma que las acciones que se lleven a cabo sobre uno no actúen de forma negativa sobre el otro. En primer lugar, la trama urbana propuesta y/o modificada debe generar una red de drenaje que dilate al máximo el tiempo de permanencia del agua y que evite la creación de 'calles río’ y ‘calles lago'. Para ello, se modificarán las pendientes de las calles que lo requieran y se forzará la creación de nuevos itinerarios de drenaje o 'corredores azules' que conducirán el agua a aquellos lugares que hayan sido diseñados para su almacenamiento, infiltración y retención (espacios verdes y áreas multifuncionales), utilizando los denominados 'interceptores de escorrentía' (Fig. 7). De esta forma se disminuirán considerablemente las áreas inundables y su frecuencia (Fig. 8).

15 Suelen aplicarse modelos hidrológicos (HEC-HMS, SWMM, MIKE, MOUSE,...) e hidráulicos (HECRAS, FESWMS-2DH,...) para el cálculo de caudales y de llanuras de inundación (Martín et al., 2003, GómezValentín et al., 2009). 
Figura 7

ELEMENTO PARA INTERCEPTAR Y DESVIAR LA ESCORRENTÍA

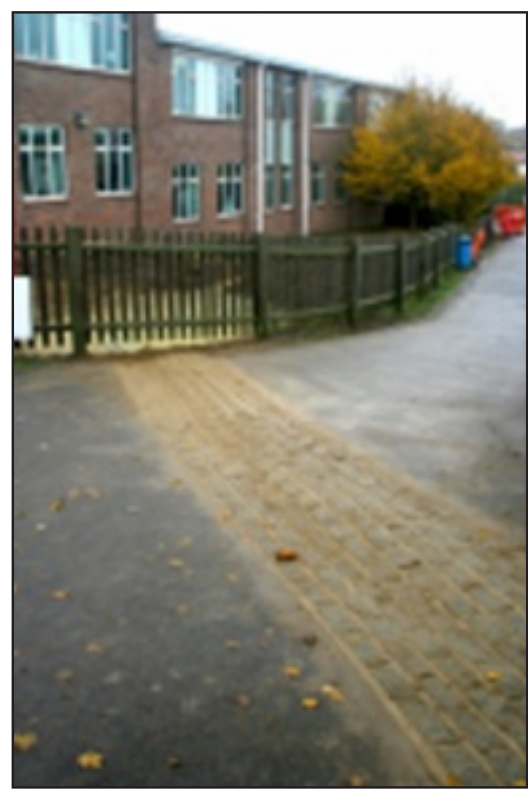

Fuente: Susdrain, 2015.

Figura 8

FASE DE PROPUESTAS

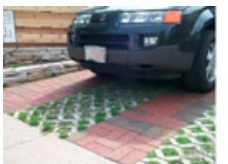

Superficie permeable
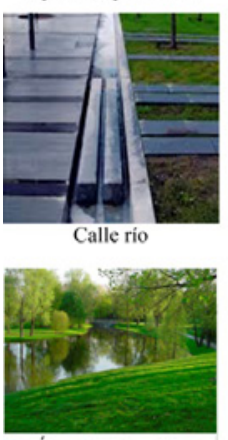

Área de retención

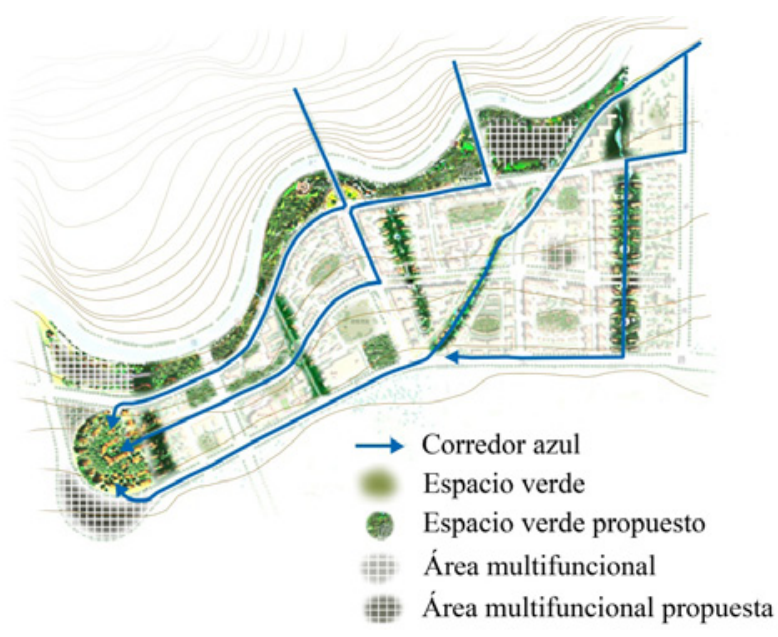

\#\# Área multifuncional propuesta

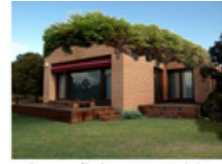

Superficie permeable

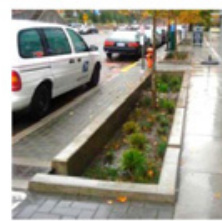

Calle lago

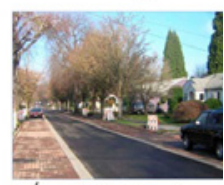

Área de infiltración

Fuente: elaboración propia. 
En segundo lugar, debe aumentarse el grado de permeabilidad de las superficies existentes y/o proyectadas mediante el rediseño de las mismas (repavimentación, incorporación de cubiertas verdes, zanjas filtrantes...), o en caso de ser necesario, llevando a cabo un cambio de uso en aquellas zonas que tengan un importante riesgo de inundación. Con ello se reducirá considerablemente la cantidad de agua que circulará por las calles, mejorando las condiciones de servicio en la ciudad y aumentando los recursos hídricos subterráneos disponibles.

Aquellos problemas que no hayan podido ser eliminados con las propuestas anteriores pueden ser minimizados mediante la integración de los SUDS en la trama urbana. Para la elección de la tipología más apropiada deben tenerse en cuenta dos variables principales; la situación del área de proyecto y el uso urbano existente. La situación condicionará la utilización de un sistema que potencie más la infiltración, la filtración, la retención o la reutilización, y el uso del suelo determinará específicamente el tipo de SUDS a emplear en función de los objetivos y beneficios perseguidos por el sistema. Tras realizar una revisión de los principales manuales técnicos de referencia (CIRIA, 2007; CIRIA, 2010; Melbourne Water, 2010; SWD, 2012; EPA, 2014; SUSDRAIN, 2015), a continuación se presenta una síntesis de la información necesaria para elegir el SUDS más adecuado en función de la situación (Cuadro 1) y del uso del suelo (Cuadro 2), facilitando así la labor del planificador.

Por último y para asegurar el éxito de las medidas propuestas, es necesario desarrollar un plan de mantenimiento que implique a los usuarios afectados por las propuestas, asegurando la conservación y mantenimiento de los sistemas que lo requieran.

1. En las partes altas y medias de la ciudad deben utilizarse SUDS que fundamentalmente retengan e infiltren el agua pluvial, con el fin de reducir la cantidad y velocidad de la escorrentía que llega a las zonas bajas.

2. En las partes bajas de la ciudad deben implantarse sobre todo sistemas que infiltren el agua sobrante de la cuenca, con el fin de disminuir la frecuencia y gravedad de las inundaciones.

3. En aquellos lugares que se encuentren degradados y que tengan problemas de contaminación, deben utilizarse SUDS específicos para mejorar la calidad del paisaje y depurar el agua pluvial.

4. En todos los casos deben buscarse alternativas para reutilizar el agua infiltrada y retenida en usos no consuntivos o en la recarga de acuíferos, aumentando la cantidad de recursos disponibles

Fuente: elaboración propia. 
Cuadro 2

INTEGRACIÓN DE LOS SUDS EN EL MEDIO URBANO EN FUNCIÓN DEL USO URBANO

\begin{tabular}{|c|c|c|c|c|c|}
\hline USO & SUDS & DESCRIPCIÓN DEL ELEMENTO & EJEMPLO & OBJ. & BENEFICIOS \\
\hline \multirow{6}{*}{$\begin{array}{c}\text { Uso } \\
\text { Residencial }\end{array}$} & $\begin{array}{l}\text { Cubierta } \\
\text { verde }\end{array}$ & $\begin{array}{l}\text { Cubierta con vegetación que retiene y } \\
\text { depura el agua de lluvia, disminuyendo } \\
\text { la temperatura del edificio y } \\
\text { posibilitando su reutilización en usos no } \\
\text { consuntivos. }\end{array}$ & & & \\
\hline & $\begin{array}{l}\text { Depósito } \\
\text { de agua de } \\
\text { lluvia }\end{array}$ & $\begin{array}{l}\text { Elemento que recolecta el agua de lluvia } \\
\text { procedente de las cubiertas para su } \\
\text { reutilización en usos no consuntivos. }\end{array}$ & & & \\
\hline & $\begin{array}{l}\text { Jardín de } \\
\text { lluvia }\end{array}$ & $\begin{array}{l}\text { Elemento vegetado adosado a los } \\
\text { edificios que retiene, trata e infiltra } \\
\text { el agua de lluvia procedente de las } \\
\text { cubiertas regenerando el paisaje. }\end{array}$ & & & \\
\hline & $\begin{array}{l}\text { Pavimento } \\
\text { modular }\end{array}$ & $\begin{array}{l}\text { Superficie permeable que retiene } \\
\text { e infiltra la escorrentía a través del } \\
\text { espacio entre piezas, disminuyendo } \\
\text { la temperatura del suelo y los } \\
\text { encharcamientos. }\end{array}$ & & & \\
\hline & $\begin{array}{l}\text { Zanja de } \\
\text { infiltración }\end{array}$ & $\begin{array}{l}\text { Zanja de material granular que infiltra } \\
\text { la escorrentía procedente de pavimentos } \\
\text { impermeables, disminuyendo } \\
\text { la temperatura del suelo y los } \\
\text { encharcamientos. }\end{array}$ & & & \\
\hline & $\begin{array}{l}\text { Pozo de } \\
\text { infiltración }\end{array}$ & $\begin{array}{l}\text { Estructura subterránea vertical de } \\
\text { material granular que infiltra la } \\
\text { escorrentía procedente de las cubiertas, } \\
\text { disminuyendo la temperatura del suelo y } \\
\text { los encharcamientos. }\end{array}$ & & & \\
\hline \multirow{3}{*}{ Calle } & $\begin{array}{c}\text { Jardín de } \\
\text { lluvia }\end{array}$ & $\begin{array}{l}\text { Elemento vegetado insertado en la acera } \\
\text { que retiene, trata e infiltra la escorrentía } \\
\text { procedente de la calzada, disminuyendo } \\
\text { la temperatura del entorno y regenerando } \\
\text { el paisaje. }\end{array}$ & & II & \\
\hline & $\begin{array}{l}\text { Arroyo } \\
\text { urbano }\end{array}$ & $\begin{array}{l}\text { Canal vegetado abierto que transporta } \\
\text { la escorrentía hacia las zonas de } \\
\text { infiltración, disminuyendo la temperatura } \\
\text { del entorno y regenerando el paisaje. }\end{array}$ & & & \\
\hline & $\begin{array}{c}\text { Acequia } \\
\text { urbana }\end{array}$ & $\begin{array}{l}\text { Canal abierto que transporta la } \\
\text { escorrentía hacia las zonas de } \\
\text { infiltración, disminuyendo la temperatura } \\
\text { del entorno y regenerando el paisaje. }\end{array}$ & $\lim ^{2}$ & 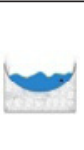 & r \\
\hline
\end{tabular}




\begin{tabular}{|c|c|c|c|c|c|}
\hline USO & SUDS & DESCRIPCIÓN DEL ELEMENTO & EJEMPLO & OBJ. & BENEFICIOS \\
\hline Calle & $\begin{array}{l}\text { Pavimento } \\
\text { modular }\end{array}$ & $\begin{array}{l}\text { Superficie permeable que retiene } \\
\text { e infiltra la escorrentía a través del } \\
\text { espacio entre piezas, disminuyendo } \\
\text { la temperatura del suelo y los } \\
\text { encharcamientos. }\end{array}$ & & & \\
\hline $\begin{array}{c}\text { Calzada y } \\
\text { Parking }\end{array}$ & $\begin{array}{l}\text { Zanja de } \\
\text { infiltración }\end{array}$ & $\begin{array}{l}\text { Zanja de material granular que infiltra } \\
\text { la escorrentía procedente de la calzada y } \\
\text { aparcamientos recargando acuíferos. }\end{array}$ & & & \\
\hline \multirow{4}{*}{$\begin{array}{c}\text { Calzada y } \\
\text { Parking }\end{array}$} & $\begin{array}{l}\text { Cuneta } \\
\text { verde }\end{array}$ & $\begin{array}{l}\text { Cuneta vegetada que transporta, infiltra } \\
\text { y trata la escorrentía procedente de la } \\
\text { calzada y aparcamientos, recargando } \\
\text { acuíferos y regenerando el paisaje. }\end{array}$ & & & \\
\hline & $\begin{array}{l}\text { Área } \\
\text { de bio- } \\
\text { retención }\end{array}$ & $\begin{array}{l}\text { Depresión vegetada del terreno } \\
\text { que transporta, infiltra y trata la } \\
\text { escorrentía procedente de la calzada y } \\
\text { aparcamientos, recargando acuíferos y } \\
\text { regenerando el paisaje. }\end{array}$ & & & \\
\hline & $\begin{array}{c}\text { Pavimento } \\
\text { drenante }\end{array}$ & $\begin{array}{l}\text { Superficie porosa que retiene e infiltra } \\
\text { la escorrentía a través de sus huecos, } \\
\text { disminuyendo la temperatura del suelo y } \\
\text { los encharcamientos. }\end{array}$ & & & \\
\hline & $\begin{array}{l}\text { Pavimento } \\
\text { modular }\end{array}$ & $\begin{array}{l}\text { Superficie modular que retiene e infiltra } \\
\text { la escorrentía a través del espacio entre } \\
\text { piezas, disminuyendo la temperatura del } \\
\text { suelo y los encharcamientos. }\end{array}$ & & & \\
\hline \multirow{3}{*}{$\begin{array}{l}\text { Espacios } \\
\text { abiertos }\end{array}$} & $\begin{array}{l}\text { Canal } \\
\text { verde }\end{array}$ & $\begin{array}{l}\text { Canal vegetado que transporta, infiltra y } \\
\text { trata la escorrentía procedente de zonas } \\
\text { impermeables de la ciudad, recargando } \\
\text { acuíferos y regenerando el paisaje. }\end{array}$ & & & \\
\hline & $\begin{array}{l}\text { Dren } \\
\text { filtrante }\end{array}$ & $\begin{array}{l}\text { Zanja de material granular que infiltra } \\
\text { la escorrentía procedente de zonas } \\
\text { impermeables de la ciudad recargando } \\
\text { acuíferos. }\end{array}$ & & It & \\
\hline & $\begin{array}{l}\text { Área de } \\
\text { infiltración }\end{array}$ & $\begin{array}{l}\text { Depresión vegetada del terreno que } \\
\text { infiltra y trata la escorrentía procedente } \\
\text { de las superficies impermeables de } \\
\text { la ciudad, recargando acuíferos y } \\
\text { regenerando el paisaje. }\end{array}$ & & 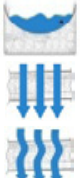 & \\
\hline
\end{tabular}




\begin{tabular}{|c|c|c|c|c|c|}
\hline USO & SUDS & DESCRIPCIÓN DEL ELEMENTO & EJEMPLO & OBJ. & BENEFICIOS \\
\hline \multirow{2}{*}{$\begin{array}{c}\text { Espacios } \\
\text { abiertos }\end{array}$} & $\begin{array}{l}\text { Área de } \\
\text { retención }\end{array}$ & $\begin{array}{l}\text { Estanque artificial que almacena } \\
\text { temporalmente la escorrentía procedente } \\
\text { de las superficies impermeables de la } \\
\text { ciudad y la libera lentamente. }\end{array}$ & & W| & \\
\hline & Humedal & $\begin{array}{l}\text { Estanque de retención e infiltración } \\
\text { natural específico para tratar la } \\
\text { escorrentía procedente de las superficies } \\
\text { impermeables de la ciudad mediante } \\
\text { vegetación natural. }\end{array}$ & & & \\
\hline Otros & Glorietas & $\begin{array}{l}\text { Depresión vegetada del terreno que } \\
\text { infiltra y trata la escorrentía procedente } \\
\text { de las infraestructuras viarias, recargando } \\
\text { acuíferos y regenerando el paisaje. }\end{array}$ & & $\begin{array}{l}\text { WH } \\
\text { W| }\end{array}$ & \\
\hline \multicolumn{6}{|c|}{ OBJETIVOS: INFILTRACIÓN } \\
\hline \multicolumn{6}{|c|}{$\begin{array}{l}\text { BENEFICIOS: CONTRIBUCIÓN A LA SOSTENIBILIDAD } \\
\text { BENEFICIOS PARA LA COMUNIDAD }\end{array}$} \\
\hline
\end{tabular}

Fuente: elaboración propia.

\section{II.4. Evaluación}

Una vez definidas las propuestas y antes de la ejecución de las mismas, es importante hacer una presentación y discusión ante los agentes sociales, políticos y técnicos involucrados en el proceso, con el fin de confirmar que todos los implicados son favorables a la ejecución de los proyectos propuestos y al plan de mantenimiento de los mismos. En caso contrario, deberán revisarse las propuestas con el fin de hacerlas compatibles con las necesidades de todos los afectados por el plan.

Por otro lado, es conveniente valorar los resultados obtenidos cuantificando la mejora que las propuestas realizadas han generado respecto de la situación previa al plan en proyectos de renovación urbana, y/o del diseño convencional que se llevaría a cabo en los nuevos desarrollos. Para ello se deberá calcular:

- La disminución del riesgo de inundación lograda (aplicando de nuevo el modelo hidrológico-hidráulico utilizado en la fase de análisis).

- La reducción del volumen total de escorrentía en la trama urbana y la cantidad de agua reutilizada en los diferentes usos.

- El incremento de superficies permeables y de espacios verdes proyectados.

- El descenso de la temperatura del medio urbano producido. 
Con esta información podrá realizarse un análisis coste-beneficio de las propuestas realizadas que podría implementarse en la viabilidad económica del plan y en su programa de amortización. Así, las autoridades podrían considerar la creación de incentivos económicos y fiscales en función de las mejoras producidas, ya que éstas se traducirán en una reducción generalizada de costes; menor mantenimiento y explotación de las redes de agua, disminución de daños e indemnizaciones en caso de inundaciones, reducción del consumo de agua y energía... Estas medidas servirían sin duda para potenciar la integración del DUSA en el planeamiento, ayudando a concienciar a todas las partes de los beneficios de la implementación de este modelo.

\section{II.5. Monitorización}

La última etapa de la metodología consiste en la evaluación periódica de los resultados obtenidos, de forma que se asegure el correcto funcionamiento de los diseños propuestos y la consecución de los objetivos planteados por el plan. Para ello, deben re-evaluarse los resultados alcanzados y en caso de ser necesario, establecer las medidas oportunas para evitar la disminución del rendimiento de los sistemas implementados y la efectividad de las propuestas realizadas.

\section{RESULTADOS}

La revisión realizada en este artículo sobre la problemática ocasionada por la impermeabilización del suelo y sus impactos, confirma la necesidad de llevar a cabo un cambio de paradigma en la gestión del drenaje urbano en el que la planificación urbana se consolide como una herramienta para mejorar la sostenibilidad en la ciudad. Para ello, se ha propuesto una metodología de trabajo cuyo principal objetivo es integrar los principios del 'diseño urbano sensible al agua' (DUSA) en la planificación (Fig. 5), de forma que se eliminen y/o mitiguen en origen los impactos generados por el sellado del suelo. En este sentido, también se han establecido unos criterios de diseño y planificación que incluyen la integración de los 'sistemas urbanos de drenaje sostenible' (SUDS) en el ámbito urbano, facilitando a los planificadores la tarea de su implementación de forma que los resultados globales obtenidos sean los más satisfactorios para el conjunto de la ciudad (Cuadros 1 y 2). Con la aplicación de esta metodología se favorecerá una forma de ocupación del territorio más sostenible y acorde con los procesos hidrológicos y se utilizará el diseño de los elementos urbanos (calles, edificios, espacios públicos, aparcamientos,...) como una herramienta para restaurar la calidad ambiental de la ciudad.

Por otro lado, la implementación de este modelo en la planificación supondrá un avance respecto de la situación actual, pues existen aún muy pocas referencias que trabajen en la definición y concreción del DUSA, centrándose la mayoría de ellas en la utilización de los SUDS como medida para resolver problemas existentes. La aplicación del 'diseño urbano sensible al agua' está generando numerosos beneficios para la ciudad a corto y medio plazo, pero también está presentando algunas dificultades para su implantación en relación a la coordinación necesaria entre las autoridades gestoras del agua y el suelo, lo cual está suponiendo una limitación en la aplicación de este modelo. Ésta es sin duda la principal debili- 
dad de la metodología planteada, pues requiere de una coordinación entre administraciones que permita que las propuestas desarrolladas respondan tanto a los requerimientos urbanos como a los hídricos. Este problema podría resolverse mediante la definición de una serie de documentos que fueran comunes en los planes territoriales e hidrológicos, obligando a que las estrategias de ambos planes actuaran de forma solidaria sobre los recursos agua y suelo. La metodología propuesta puede resultar muy útil en esta tarea, ya que concreta las variables que sería necesario considerar para llevar a cabo la integración, así como los principios que deberían tenerse en cuenta en la redacción de estos documentos.

\section{CONCLUSIONES}

El crecimiento de las ciudades en el último siglo ha generado un olvido generalizado del ciclo del agua que ha ocasionado impactos sociales, económicos y ambientales muy graves, sobre todo en relación al agua. La continua impermeabilización de los suelos ha generado un aumento de los volúmenes de escorrentía que las redes de saneamiento no han podido absorber, a pesar de haber sido ampliadas de forma continua a lo largo de los últimos años. Esto ha generado un modelo de gestión del drenaje insostenible que está demandando una nueva forma de tratar el agua pluvial en la ciudad más acorde con los principios medioambientales. Para ello en los últimos años se están utilizando los SUDS en muchos países del mundo como instrumento para recuperar en la medida de lo posible el sentido natural del ciclo hidrológico en la ciudad, a través de la infiltración, filtración, retención y reutilización del agua pluvial. A pesar de haber supuesto un avance importante en la búsqueda de formas más sostenibles de gestionar este recurso, en la mayoría de ocasiones estos sistemas se han implementado sólo para minimizar a posteriori los impactos generados por el sellado del suelo. Dado que el origen de estos problemas se encuentra en el modelo de ocupación urbana actual, se ha comenzado a plantear la necesidad de desarrollar una nueva estrategia de planeamiento denominada 'diseño urbano sensible al agua' y cuyo objetivo principal es implementar los requerimientos del agua en la planificación de las ciudades. De esta forma, se podrán eliminar y/o mitigar los problemas generados por la impermeabilización del suelo, generando un modelo urbano más sostenible que utiliza el agua pluvial como una herramienta para la revalorización del entorno urbano y la mejora de la calidad paisajística y ambiental de la ciudad. Así, el ciudadano sufrirá en menor medida las graves consecuencias de las inundaciones, las empresas gestoras del saneamiento urbano verán disminuidos sus costes de explotación y renovación y las autoridades municipales podrán asegurar mejores condiciones de servicio en la ciudad. A lo largo de este artículo ha quedado patente que el planeamiento urbano debe ser el marco de referencia en el que debe integrarse este nuevo concepto, no como algo aislado, sino como parte de un proceso de planificación basado en la recuperación del ciclo natural del agua. La ausencia de referencias teóricas en relación al DUSA hace necesario desarrollar guías específicas dirigidas a urbanistas y planificadores que faciliten la aplicación de este modelo. En este sentido, este artículo propone una herramienta metodológica que integra los requerimientos del DUSA en el proceso de planificación, contribuyendo al desarrollo de proyectos sensibles al agua, a la mejora de la calidad ambiental de las ciudades y al aumento de la sostenibilidad de la gestión del recurso hídrico. 


\section{REFERENCIAS}

ANTROP, M. (2004): «Landscape change and the urbanization process in Europe». Landscape and Urban Planning, $\mathrm{n}^{\circ}$ 64, 9-26.

ARNFIELD, A. J. (2003): «Two decades of urban climate research: a review of turbulence, exchanges of energy and water, and the urban heat island». International Journal of Climatology, $\mathrm{n}^{\mathrm{o}} 23(1), 1-26$.

ASLA (2015). www.asla.org. Consultado el 23/03/2015.

BENGSTON, D.N., FLETCHER, J.O. y NELSON, K.C. (2004): «Public policies for managing urban growth and protecting open space: policy instruments and lessons learned in the United States». Landscape and Urban Planning, ${ }^{\circ}$ 69, 271-286.

BLUM,W.E.H., BÜSING, J. y MONTANARELLA, L. (2004): «Research needs in support of the European thematic strategy for soil protection». Trac.TrendsAnal.Chem. $\mathrm{n}^{\circ} 23$, 680-685.

BOLUND P. y HUNHAMMAR S. (1999): «Ecosystem services in urban areas». Ecological Economics, n' $29,293-301$.

BURGHARDT, W. FORMEN UND WIRKUNG DER VERSIEGELUNG. (1993): Symposium Bodenschutz Zentrum far Umweltforschung der Westfiilischen Wilhelms Universitiit, pp. 111-125.

BURGHARDT, W., BANKO, G., HOEKE, S., HURSTHOUSE, A., DE L'ESCAILLE, TH., LEDIN, ST., MARSAN, F.A., SAUER, D., STAHR, K., AMANN, E., QUAST, J., NERGER, M., SCHNEIDER, J. y KUEHN, K., (2004): «Sealing soils, soils in urban areas, land use and land use planning». En Report of Task Group 5, Vol. VI. Research, sealing and cross-cutting issues.

CARSJENS, G.J. y VAN LIER, H.N. (2002): «Fragmentation and Land-Use Planning - An introduction». Landscape and Urban Planning, $\mathrm{n}^{\circ}$ 58, 79-82.

CATALÁ, F. (1992): Cálculo de caudales en las redes de saneamiento. Colegio de Ingenieros de Caminos, Canales y Puertos. Colección Senior n ${ }^{\circ}$ 5. Madrid.

CERDÁ I. (1867): Teoría general de la urbanización y aplicación de sus principios y doctrinas a la reforma y ensanche de Barcelona. Reeditado por el Instituto de Estudios Fiscales, Barcelona.

CHEN, Z., CHEN, J., SHI, P. y TAMURA, M. (2003): «An HIS based change detection approach for assessment of urban expansion impact on arable land loss in China». International Journal of Remote Sensing, $\mathrm{n}^{\circ} 24$ (6), 1353-1360.

CIRIA C521 (2000): Sustainable urban drainage systems, design manual for Scotland and Nothern Ireland. CIRIA, London.

CIRIA C697 (2007): The SUDS Manual. CIRIA, London.

CIRIA C687 (2010): Planning for SUDS-making it happen. CIRIA, London.

COAG (2004); Council of Australian Governments' Meeting . Celebrado en Canberra 25 Junio, 2004.Disponible en http://archive.coag.gov.au/coag_meeting_outcomes/2004-06-25/ index.cfm

CONESA, C. y GARCÍA, E. (2003): «Las áreas históricas de inundación en Cartagena: problemas de drenaje y actuaciones». Boletín de la Asociación de Geógrafos Españoles, $\mathrm{n}^{\mathrm{o}} 35,79-100$. 
DIRECTION DE L'URBANISME ET DES PAYSAGES (1982): La maîtrise du ruissellement des eaux pluviales. Ministère de L'Urbanisme et du Logement. Paris.

EEA (2006): Urban sprawl in Europe - The ignored challenge. European Environment Agency, Report No 10/2006. Copenhagen, 2006.

EEA (2010): The European environment - State and Outlook 2010: land use. European Environment Agency, Copenhagen.

ELSAYED, I. S. M., (2012): «Mitigation of the Urban Heat Island of the City of Kuala Lumpur», Malaysia, n 11(11), 1602-1613.

EPA (2008): Reducing Urban Heat Island: Compendium of Strategies. Disponible en http:// www.epa.gov/heatisland/resources/compendium.htm. Consultado el 13/09/2015.

EPA (2014) http://www.epa.gov. consultado el 24/11/2014

FERRAS, C., (2000): «Innovación, desarrollo y medio local. Dimensiones sociales y espaciales de la innovación». Scripta Nova, Revista Electrónica de Geografía y Ciencias Sociales, $\mathrm{n}^{\circ} 69$ (68).

GARCÍA, P., PÉREZ, M. E. y GUERRA, A. (2014): «Using TM images to detect soil sealing change in Madrid (Spain). Geoderma, $\mathrm{n}^{\circ} 214,135-140$.

GARCÍA, J.C. (2004): «Las ciudades españolas y el riesgo de inundación: permanencia y cambio de un problema crónico». Boletín de la Asociación de Geógrafos Españoles, ${ }^{\circ}$ 37, 85-99.

GARTLAND, L. (2008): «Heat Islands: Understanding and Mitigating Heat in Urban Areas. Earthscan Publications, 57-83.

GIBB, H. y HOCHULI, D.F. (2002): «Habitat fragmentation in a urban environment: large and small fragments support different arthropod assemblages». Biological Conservation, $\mathrm{n}^{\mathrm{o}} 106,91-100$.

GOBSTER, P.H., STEWART, S.I. y BENGSTON, D.N. (2004): «The social aspects of landscape change: protecting open space under the pressure of development». Landscape and Urban Planning, nº 69 (2-3), 149-151.

GÓMEZ-VALENTÍN, M.; MACCHIONE, F.; RUSSO, B. (2009): «Comportamiento hidráulico de las calles durante lluvias extremas en zonas urbanas». Ingeniería Hidráulica en México, $\mathrm{n}^{\mathrm{o}} 24$ (3), 51-62.

HAUSMANN, M. (2014). Blog personal. Los techos verdes y las fotografías de MacLean. Disponible en http://maxhausmannmtz.wordpress.com/2013/08/06/alex-mclean-verde/

HOUŠKOVÁ, B. y MONTANARELLA, L. (2008): «The natural susceptibility of european soils to compaction. Threats to Soil Quality in Europe. European Commission, Joint Research Centre, Institute for Environment and Sustainability, pp. 23-36, 2008.

IMHOFF, M.L., BOUNOUA, L., DEFRIES, R., LAWRENCE, W.T., STUTZER, D., TUCKER, C.J. y RICKETTS, T. (2004): «The consecuences of urban land transformation on net primary productivity in the United States». Remote Sensing of Environment, ${ }^{\circ} 89$ (4), 434-443.

LAVALLE, C., DEMICHELI, L., KASANKO, M., TURCHINI, M., NIEDERHUBER, M. y MCCORMICK N., (2001): Murbandy/Moland technical report. European Commission Euro-Report. 
LAWRENCE, W.T., IMHOFF, M.L., KERLE, N. y STUTZER, D. (2002): «Quantifying urban land use and impact on soils in Egypt using diurnal satellite imagery of the Earth surface». International Journal of Remote Sensing, $\mathrm{n}^{\circ} 23$ (19), 3921-3937.

MARTÍN, J.P., LÓPEZ, S., MARTÍN, P., SIMARRO, G., BENITO, G. (2003): «USO DE MODELOS UNI- Y BIDIMENSIONALES EN LLANURAS DE INUNDACIÓN. APLICACIÓN AL CASO DEL RÍO TAJO EN TALAVERA DE LA REINA». Ingeniería del agua, $\mathrm{n}^{\circ} 10(1), 49-58$.

MELBOURNE WATER (2010): WSUD Guidelines. An Initiative of the Inner Melbourne Action Plan. Melbourne water. Sydney.

MINVU-DICTUC (1996): Técnicas alternativas para soluciones de aguas lluvias en sectores urbanos. Guía de diseño. Dirección de Investigaciones Científicas y Tecnológicas. Ministerio de Vivienda y Urbanismo. Santiago, Chile.

MONCLÚS, J. (ed.) (1998): La ciudad dispersa. Centro de Cultura Contemporánea, Barcelona.

MONTANARELLA, L., (2007): «Trends in land degradation in Europe». Sivakumar, M.V.K., Ndegwa, N. Springer-Heidelberg, Germany, 83-104.

MORELLO, J., BUZAI, G.D., BAXENDALE, A.B., RODRÍGUEZ, A.F., MATTEUCCI, S.D., GODAGNONE, R.E. y CASAS, R.R. (2000): «Urbanization and the consumption of fertile land and other ecological changes: the case of Buenos Aires». Environment and Urbanization, $\mathrm{n}^{\circ} 12$ (2), 119-131.

MWB, Melbourne Water Corporation (2013): Water Sensitive Urban Design, Melbourne.

OLSSON, J., BERGGREN, K., OLOFSSON, M. y VIKLANDER, M. (2009): «Applying climate model precipitation scenarios for urban hydrological assessment: A case study in Kalmar City, Sweden». Atmospheric Research, n 92(3), pp. 364-375.

PROKOP G., JOBSTMANN H. y SCHÖNBAUER A. (2011): Overview on best practices for limiting soil sealing and mitigating its effects in EU-27. Environment Agency Austria.

PRUD’HOMME, R. y LEE, C.W. (1999): «Size, Sprawl, Speed and the Efficiency of Cities». Urban Studies, ${ }^{\circ} 36$ (11), 1849-1858.

REBSTOCK, M., BERDING, J., GATHER, M., HUDEKOVA, Z., \& PAULIKOVA, M. (2011): Methodology Plan for good planning and designing of urban open spaces. UE, UrbSpace-Project.

REN, W., ZHONG, Y., MELIGRANA, J., ANDERSON, B., WATT, W.E., CHEN, J. y LEUNG, H.L. (2003): «Urbanization, land use and water quality in Shanghai: 19471996». Environment International, no 29 (5), 649-659.

RODRÍGUEZ-ROJAS M.I., CUEVAS M.M., MARTÍNEZ G. y MORENO B. (2014): «Planning Criteria for Water Sensitive Urban Design». WIT Transactions on Ecology and the Environment, n ${ }^{\circ} 191,1579-1591$.

RODRÍGUEZ-ROJAS M.I., CUEVAS M.M., MARTÍNEZ G. y MORENO B. (2015): «Indicators to evaluate Water Sensitive Urban Design in Urban Planning». WIT Transactions on The Built Environment, $\mathrm{n}^{\mathrm{o}} 168$ (1), 371-382.

SANTAMOURIS, M., PAPANIKOLAOU, N., LIVADA, I., KORONAKIS, I., GEORGAKIS, C., ARGIRIOU, A. y ASSIMAKOPOULOS, D. (2001): «On the impact of urban climate on the energy consumption of buildings». Solar Energy, n $70(3), 201-216$. 
SEMADENI-DAVIESA A., HERNEBRINGB C., SVENSSONB G. y GUSTAFSSONC L. (2009): «The anthropogenic sealing of soils in urban areas». Landscape and Urban Planning, no 90 (1-2), 1-10.

SIERRA CLUB, (2000): Sprawl costs Us All. How your Taxes Fuel Suburban Sprawl. Sierra Club Foundation.

SUSDRAIN (2015): www.susdrain.org. Consultado el 12/04/2015.

SWD 101, (2012): Guidelines on best practice to limit, mitigate or compensate soil sealing. Commission Staff Working Document.

TECHNISCHE COMMISSIE BODEM (2010): Advisory report on general conditions for soil sealing in urban areas. TCB A063, The Hague and references therein.

TÓTH, G., MONTANARELLA, L. $y$ RUSCO, E. (2008): Threats to soil quality in Europe, JRC publication 46574. Office for Official Publications of the European Communities, Luxembourg.

VAN-CAMP, L., BUJARRABAL, B., GENTILE, A.R., JONES, R., MONTANARELLA, L., OLAZÁBAL, C. y SELVARADJON, S.K. (2004): Reports of the technical working groups, EUR 21319 EN/6 872. Office for Official Publ. of the European Communities (Vo. VI. Luxembourg).

WSUD (2014): www.wsud.org. Consultado el 18/04/2015. 